\title{
Analysis of spontaneous MEG activity in mild cognitive impairment and Alzheimer's disease using spectral entropies and statistical complexity measures
}

\author{
Ricardo Bruña , Jesús Poza , , Carlos Gómez , María García , \\ Alberto Fernández and Roberto Hornero
}

\begin{abstract}
Alzheimer's disease (AD) is the most common cause of dementia. Over the last few years, a considerable effort has been devoted to exploring new biomarkers. Nevertheless, a better understanding of brain dynamics is still required to optimize therapeutic strategies. In this regard, the characterization of mild cognitive impairment (MCI) is crucial, due to the high conversion rate from MCI to AD. However, only a few studies have focused on the analysis of magnetoencephalographic (MEG) rhythms to characterize AD and MCI. In this study, we assess the ability of several parameters derived from information theory to describe spontaneous MEG activity from $36 \mathrm{AD}$ patients, $18 \mathrm{MCI}$ subjects and 26 controls. Three entropies (Shannon, Tsallis and Rényi entropies), one disequilibrium measure (based on Euclidean distance ED) and three statistical complexities (based on Lopez Ruiz-ManciniCalbet complexity LMC) were used to estimate the irregularity and statistical complexity of MEG activity. Statistically significant differences between AD patients and controls were obtained with all parameters $(p<0.01)$. In addition, statistically significant differences between MCI subjects and controls were achieved by ED and LMC $(p<0.05)$. In order to assess the diagnostic ability of the parameters, a linear discriminant analysis with a leave-one-out cross-validation procedure was applied. The accuracies reached $83.9 \%$ and $65.9 \%$ to discriminate AD and MCI subjects from controls, respectively. Our findings suggest that MCI subjects exhibit an intermediate pattern of abnormalities between normal aging and AD. Furthermore, the proposed parameters provide a new description of brain dynamics in AD and MCI.
\end{abstract}




\section{Introduction}

Alzheimer's disease (AD) is a neurodegenerative disorder of unknown etiology that represents the most common cause of dementia (Blennow et al 2006). Several risk factors have been identified, though age is the most important one. This is a crucial factor in developed countries, due to the increase in life expectancy (Blennow et al 2006). Early symptoms of AD include memory loss and concentration problems. AD progression is often accompanied by speech disorders, such as aphasia, apraxia and agnosia, together with general cognitive symptoms (Jalbert et al 2008). AD clinical diagnosis is achieved by means of a complete medical history and by performing physical, psychiatric and neurological examinations. Diagnosis is also supported by laboratory and imaging studies in order to exclude age-related diseases with similar symptomatology (Minati et al 2009). An optimal treatment requires intervention in early stages, even before the appearance of the first clinical symptoms (Jalbert et al 2008). Consequently, the characterization of mild cognitive impairment (MCI) arises as a crucial issue.

Structural changes in AD are related to the accumulation of amyloid plaques among nerve cells in the brain, as well as the appearance of neurofibrillary tangles inside nerve cells (Cummings 2004). These are considered as AD hallmarks. However, it is not yet known whether plaques or tangles cause $\mathrm{AD}$ or are a by-product of some other process. They are both formed by clusters of proteins accumulated in greater quantities, mainly in two specific brain regions: the hippocampus and the cerebral cortex. The appearance of these two abnormal microscopic structures produces neural damage or death, which is followed by a chemical imbalance. The most significant chemical change is a loss of the neurotransmitter acetylcholine. Thus, as damage to neurons in the brain increases, the ability to produce acetylcholine decreases. Both structural and chemical changes produce a progressive cell death and an overall shrinkage of the brain tissue, which culminates in the progressive clinical symptoms of AD (Cummings 2004).

MCI is usually considered as an intermediate stage between the cognitive decline associated with normal aging and a state of dementia (Petersen 2009). MCI can affect several brain areas, as those related to thought and action. The most common symptom is memory loss, whereas the ability of judgment and reasoning remain unaffected (Petersen et al 2009). This MCI subtype is often considered an initial stage in $\mathrm{AD}$ progression (Petersen et al 2009). Currently, there is still no clinically proven method to detect MCI. Therefore, more research is required to explore new MCI biomarkers for clinical use.

MCI and AD are cortical disorders. As a consequence, it could be hypothesized that the electromagnetic activity generated by the cortex could be altered to some extent. Electroencephalographic (EEG) and magnetoencephalographic (MEG) signals record the electromagnetic brain activity. They are generated by synchronous oscillations of pyramidal neurons, but reflect slightly different features (Rampp and Stefan 2007). MEG recordings are reference free. In addition, they are less affected by the volume conduction when compared to EEG (Stam 2010) and even some studies suggest that the effect of the volume conduction is to filter out high temporal frequencies (Nunez et al 2001). Therefore, MEG might be more sensitive to measure the cortical activity than scalp EEG (Georgopoulos et al 2007, Stam 2010).

Several MEG studies have been carried out in order to explore the differences in the spontaneous electromagnetic rhythms between healthy elders and $\mathrm{AD}$ patients (Stam 2010). Results in some of these studies indicated that AD is accompanied by an increase in low frequency power (Berendse et al 2000, Fernández et al 2006, Wan et al 2006, Poza et al 2007), a global decrease in irregularity (Poza et al 2007, 2008, Hornero et al 2009), a loss of complexity (van Cappellen van Walsum et al 2003, Gómez et al 2009a, Hornero et al 2009) and a global decrease of functional interactions (Berendse et al 2000, Stam 2005, Alonso et al 2011). Nevertheless, only a few investigations have analyzed the differences between MCI subjects and both $\mathrm{AD}$ patients and healthy elders, despite it being crucial for early detection of $\mathrm{AD}$. A slight increase in low frequency power of $\mathrm{AD}$ patients in comparison to $\mathrm{MCI}$ subjects has been found in previous studies (Fernández et al 2006, Osipova et al 2006), whereas an increase of complexity has been observed in MCI subjects using nonlinear measures (Fernández et al 2010). In addition, different patterns of connectivity between both groups have been found in studies based on functional connectivity (Escudero et al 2011). Their results suggested that $\mathrm{AD}$ increases the level of coherence in the delta band, while MCI subjects tend to have lower connectivity in theta band. The results in the upper bands were not conclusive, but a slight reduction in alpha interregional connectivity was found. On the other hand, only subtle differences have been reported between MCI and control subjects (Fernández et al 2006, 2010, Gómez et al 2009b, Escudero et al 2011). The results in these studies indicated that MCI subjects show intermediate abnormalities between AD patients and elderly controls. Further research is required for an appropriate description of specific brain dynamics associated with MCI.

In this study, we apply new parameters derived from information theory to characterize MEG rhythms in $\mathrm{AD}$ and MCI. Parameters derived from information theory provide an alternative description to conventional spectral and nonlinear measures. Thus, they have been successfully used to characterize EEG during an epileptic seizure (Rosso et al 2006). Despite their potential for studying brain dynamics, they have barely been used to analyze MEG (Poza et al 2008, Bruña et al 2010). Therefore, the aims of this study were: (i) to analyze several irregularity and complexity parameters based on a time-frequency representation, (ii) to describe the abnormalities of $\mathrm{AD}$ and $\mathrm{MCI}$ in comparison to cognitive decline in normal aging, and (iii) to introduce an alternative framework to understand brain dynamics. 


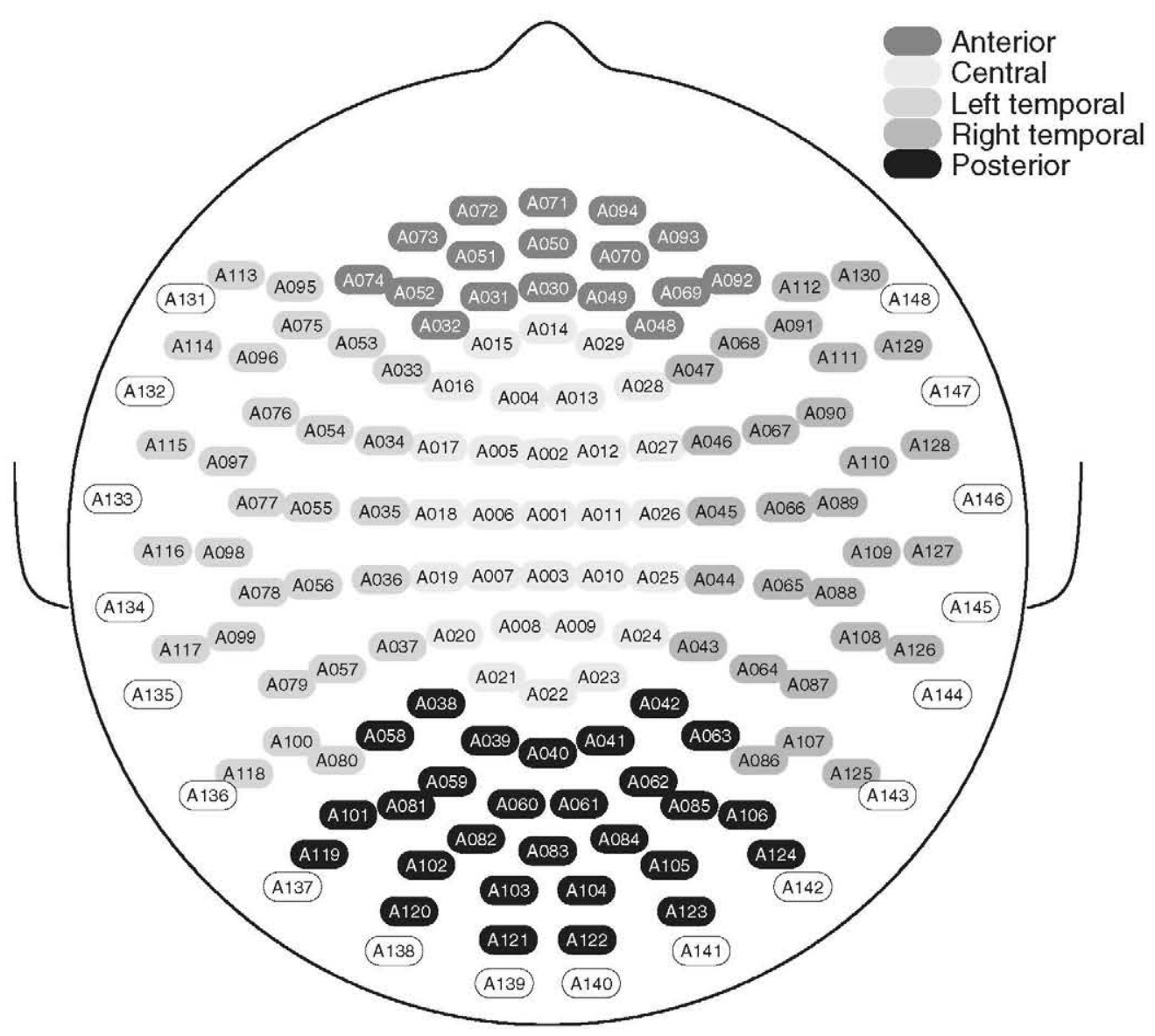

Figure 1. Sensor grouping in the 4D-Neuroimaging source analysis software, where five regions are considered for spatial analyses (anterior, central, left lateral, posterior and right lateral). Exterior channels were excluded due to the low signal to noise ratio.

\section{Materials}

\subsection{Selection of subjects}

MEG signals from eighty subjects were recorded at the 'Centro de Magnetoencefalografía Dr Pérez-Modrego' (Complutense University of Madrid, Spain). Thirty-six subjects (12 men and 24 women, age $=74.1 \pm 6.9$ years, mean \pm standard deviation $\mathrm{M} \pm \mathrm{SD}$ ) were $\mathrm{AD}$ patients derived from the 'Asociación de Familiares de Enfermos de Alzheimer' (AFAL) and the Geriatric Unit of the 'Hospital Clínico Universitario San Carlos' (Madrid, Spain). Diagnoses were made on the basis of exhaustive medical, physical, neurological, psychiatric and neuropsychological examinations, complemented with brain scans in order to exclude other causes of dementia. All patients fulfilled the criteria for probable $\mathrm{AD}$, according to the clinical guidelines of the National Institute of Neurological and Communicative Disorders and Stroke and the AD and Related Disorders Association (NINCDS-ADRDA) (McKhann et al 1984). The Mini-Mental State Examination (MMSE) and the Functional Assessment Staging (FAST) were used to evaluate the cognitive and functional deficits. $\mathrm{AD}$ patients obtained mean scores of $18.1 \pm 3.4$ and $4.2 \pm 0.4$ on the MMSE and FAST, respectively. None of these AD patients suffered from any other significant medical, neurological or psychiatric disorder.
Eighteen subjects $(8$ men and 10 women, age $=74.9 \pm$ 5.6 years, $\mathrm{M} \pm \mathrm{SD}$ ) were MCI subjects derived from AFAL. All patients were diagnosed with MCI following Petersen's criteria (Petersen et al 2001). Mean MMSE and FAST scores in this group were $25.7 \pm 1.8$ and $3.0 \pm 0.0$, respectively.

Finally, 26 healthy subjects ( 10 men and 16 women, age $=72.0 \pm 5.9$ years, $\mathrm{M} \pm \mathrm{SD}$ ) were enrolled in the study as a control group. They were cognitively normal elderly controls with no history of neurological or psychiatric disorders. Mean MMSE and FAST scores were $29.0 \pm 1.2$ and $1.7 \pm 0.5$, respectively.

None of the subjects was taking drugs that could affect MEG activity at the recording time. We did not obtain significant differences in the mean age of AD patients, MCI subjects and controls $(p>0.05$, Kruskal-Wallis test). In addition, all healthy volunteers and caregivers of patients accepted to participate in the study and gave their written informed consent. The research was approved by the Research Ethics Committee of the center.

\section{2. $M E G$ recording}

MEG signals were obtained using a 148-channel wholehead magnetometer (MAGNES 2500 WH, 4D Neuroimaging, San Diego, CA). Exterior channels were discarded due to a low signal-to-noise ratio, so that 130 channels were finally selected for analysis (see figure 1). MEG recordings 
were performed in a magnetically shielded room in order to minimize electromagnetic interferences. During data acquisition, subjects were asked to remain awake, relaxed and with their eyes closed, in order to minimize the presence of artifacts. Additionally, MEG signals were continuously monitored to prevent drowsiness. Thus, 5 min of spontaneous MEG activity were acquired with a sample frequency of $678.17 \mathrm{~Hz}$. Initially, a $0.1-200 \mathrm{~Hz}$ hardware band-pass filter and a $50 \mathrm{~Hz}$ notch filter were applied. Then, each MEG recording was downsampled by a factor of 4 to reduce the data length. Artifact-free epochs of $10 \mathrm{~s}(26.6 \pm 5.7$ artifact-free epochs per channel and subject, $\mathrm{M} \pm \mathrm{SD}$ ) were selected for further analysis. Prior to calculation of parameters, MEG signals were processed using a finite impulse response (FIR) filter designed with a Hamming window and cut-off frequencies at 1 and $70 \mathrm{~Hz}$. This frequency range was selected in order to keep the relevant spectral content and to minimize the presence of oculographic and myographic artifacts (Poza et al 2008).

\section{Methods}

\subsection{Time-frequency representation}

Electromagnetic brain recordings are non-stationary signals. Therefore, non-stationary signal analysis techniques, such as the short-time Fourier transform (STFT), are required to characterize their spectral time-varying properties. The STFT is based on the sliding temporal window technique and has been successfully applied to characterize MEG activity in AD (Poza et al 2008). In the present work, each MEG recording of $10 \mathrm{~s}$ ( $M=1696$ samples) has been divided into nonoverlapping time windows of $0.5 \mathrm{~s}$. The window length is denoted by $L$ ( $L=84$ samples) and each time interval is identified by superindex $i\left(i=1, \ldots, N_{T}\right.$, with $\left.N_{T}=M / L\right)$. Then, the temporal evolution of the power spectral density (PSD) was calculated as the Fourier transform (FT) of the autocorrelation function in each time window (Poza et al 2008). Finally, the spectral content between 1 and $70 \mathrm{~Hz}$ was selected and the PSD was normalized to scale from 0 to 1 , leading to the normalized PSD $\left(\mathrm{PSD}_{n}\right)$,

$$
\operatorname{PSD}_{n}^{(i)}(f)=\frac{\operatorname{PSD}^{(i)}(f)}{\sum_{f^{\prime}=1 \mathrm{~Hz}}^{70 \mathrm{~Hz}} \operatorname{PSD}^{(i)}\left(f^{\prime}\right)}, i=1, \ldots, N_{T} .
$$

After the normalization, it follows that $\sum_{f^{\prime}=1 \mathrm{~Hz}}^{70 \mathrm{~Hz}} \operatorname{PSD}_{n}^{(i)}\left(f^{\prime}\right)=$ 1 , for each time interval $i$. Thus, the $\operatorname{PSD}_{n}^{(i)}(f)$ can be considered as a time-varying ( $N_{T}$ time intervals) probability density function, which will be used to define the following parameters.

\subsection{Spectral entropies}

The irregularity measures analyzed in this work are Shannon (SSE), Tsallis (TSE) and Rényi (RSE) spectral entropies. Shannon entropy is a disorder quantifier, whose original meaning was related to the uncertainty in a flux of information, in terms of disorder, discrepancy or diversity (Bezerianos et al 2003). The irregularity is estimated in terms of the flatness of the $\operatorname{PSD}_{n}$. Thus, a highly irregular signal with a flat spectrum (e.g. white noise) takes a high SSE value, whereas a very predictable signal (e.g. a sum of sinusoids) takes a low SSE value (Inouye et al 1991). In the present study, the SSE definition is based on Shannon entropy computed over the time evolution of the $\mathrm{PSD}_{n}$,

$$
\begin{aligned}
\mathrm{SSE}^{(i)} & =-\sum_{f=1 \mathrm{~Hz}}^{70 \mathrm{~Hz}} \operatorname{PSD}_{n}^{(i)}(f) \cdot \ln \left[\operatorname{PSD}_{n}^{(i)}(f)\right], \\
i & =1, \ldots, N_{T} .
\end{aligned}
$$

Tsallis entropy is a generalized information measure, which extends the notion of Shannon entropy by means of a new non-logarithmic entropic formalism (Tsallis 1988). Tsallis entropy is a non-extensive measure, whose degree of non-extensivity is controlled by the entropic index $q \in \mathbb{R}$ (Tsallis 1988). Boltzmann-Gibbs entropy definition can be recovered in the limit $q \rightarrow 1$ (Tsallis 1988). The TSE definition based on the time evolution of the $\mathrm{PSD}_{n}$ can be seen in the following equation:

$$
\begin{aligned}
& \operatorname{TSE}^{(i)}(q)=\frac{1}{q-1} \sum_{f=1 \mathrm{~Hz}}^{70 \mathrm{~Hz}}\left\{\operatorname{PSD}_{n}^{(i)}(f)-\left[\operatorname{PSD}_{n}^{(i)}(f)\right]^{q}\right\}, \\
& q>0, i=1, \ldots, N_{T} .
\end{aligned}
$$

Rényi entropy is also a generalized information measure. Similar to Shannon entropy, it is an additive entropy, though Rényi entropy introduces a new logarithmic entropic formalism (Rényi 1970). Analogously to TSE, RSE is parameterized by an entropic index $q \in \mathfrak{R}$ and can be reduced to Boltzmann-Gibbs entropy in the limit $q \rightarrow 1$ (Rényi 1970). The RSE definition computed over the time evolution of the $\mathrm{PSD}_{n}$ is given by

$$
\begin{aligned}
& \operatorname{RSE}^{(i)}(q)=\frac{1}{1-q} \ln \left\{\sum_{f=1 \mathrm{~Hz}}^{70 \mathrm{~Hz}}\left[\operatorname{PSD}_{n}^{(i)}(f)\right]^{q}\right\}, \\
& q>0, i=1, \ldots, N_{T} .
\end{aligned}
$$

It is noteworthy that in this study SSE, TSE and RSE were normalized to $[0-1]$ by dividing the previous definitions by the maximum entropy values of $\ln (R),\left[1-R^{(1-q)}\right]$ and $\ln (R)$, respectively (where $R$ represents the number of frequency bins) (Bezerianos et al 2003, Rosso et al 2006).

The role of the entropic index $q$ in the parameterized entropies (TSE and RSE) was discussed and found to be relevant in a previous study (Poza et al 2008). According to the reported findings, we use $q=2$ and $q=3.5$ for TSE and RSE, respectively.

\subsection{Disequilibrium}

In order to provide an alternative framework for quantifying the irregularity of MEG recordings, a disequilibrium measure was used in the present study. The disequilibrium is defined as the distance, in the probability space, between the uniform distribution and the distribution of the data under study (LópezRuiz et al 1995). It is noteworthy that the uniform distribution is considered as the 'equilibrium' distribution in Gibbs' statistical mechanics (Martin et al 2003). As a consequence, 
a highly irregular signal like the white noise yields a low disequilibrium value. In contrast, the disequilibrium is high for a highly regular signal. Although the distance in a probability space can be quantified using several definitions, a previous study showed that the Euclidean distance (ED) could be appropriate to characterize MEG activity from MCI subjects (Bruña et al 2010). The definition of disequilibrium used in the present study is

$\mathrm{ED}^{(i)}=-\sum_{f=1 \mathrm{~Hz}}^{70 \mathrm{~Hz}}\left[\operatorname{PSD}_{n}^{(i)}(f)-1 / L\right]^{2}, \quad i=1, \ldots, N_{T}$

As in the case of spectral entropies, the ED was normalized to take values in the $0-1$ interval. Hence, equation (5) was divided by its maximum, $(R-1) / R$ (Martin et al 2003).

\subsection{Complexity measures}

Finally, a set of statistical complexity measures was proposed in the present study to capture the interplay between the previous parameters, i.e. entropies and disequilibrium. Lopez Ruiz-Mancini-Calbet (LMC) statistical complexity is a quantifier of the degree of physical structure in a time series (López-Ruiz et al 1995). This measure considers that a statistical distribution has two extreme states: a complete foreknowledge of the system (highly ordered) and a total uncertainty (highly disordered). LMC complexity assumes that both extreme states (perfect order and maximal randomness) can be regarded as trivial ones. Therefore, LMC complexity must be zero for those two extremes, but it must reach a maximum at some intermediate point (Rosso et al 2006). It is noteworthy that LMC complexity has been previously applied to EEG signals in order to analyze different brain states during an epileptic seizure (Rosso et al 2006). Their results showed that the statistical complexity yielded a complementary description of the system dynamics to that provided by irregularity measures (Rosso et al 2006). In this study, the definition of LMC complexity is based on a combination of two different quantities: an entropy quantifier and a disequilibrium measure, both computed over the time evolution of the $\mathrm{PSD}_{n}$,

$$
\mathrm{LMC}_{\mathrm{ED}, E}^{(i)}=\mathrm{ED}^{(i)} \cdot \mathrm{SE}_{(E)}^{(i)}, \quad i=1, \ldots, N_{T},
$$

where $\mathrm{ED}^{(i)}$ is the time evolution of the disequilibrium measure based on $\mathrm{ED}$, and $\mathrm{SE}_{(E)}^{(i)}$ represents the time evolution of the spectral entropies previously defined, with $E=\{\operatorname{SSE}, \operatorname{TSE}(q)$, $\operatorname{RSE}(q)\}$.

\subsection{Statistical analysis}

Initially, an exploratory analysis was carried out to study the data distribution. After the descriptive analysis, logtransformed variables met parametric test assumptions. Five brain areas were defined to group the data (anterior, central, left lateral, posterior and right lateral; see figure 1), according to the default sensor grouping in the 4D-Neuroimaging source analysis software (Poza et al 2008, Alonso et al 2011). Averaged entropy, disequilibrium and complexity values in these regions were compared between $\mathrm{AD}$ patients, MCI subjects and controls by means of two-way repeated measures ANOVAs (with 'group' as between-subject factor and 'region' as within-subject factor). Univariate ANOVAs with contrasts were performed when previous analyses showed significant interactions, using Tukey's HSD (Honestly Significant Difference) correction for discrimination between more than two groups. To correct any possible violation of the sphericity assumption and to reduce type I errors, GreenhouseGeisser epsilon was used in all repeated measures analyses. Differences were considered statistically significant for $p<0.05$.

The classification performance of each parameter was evaluated using a linear discriminant analysis (LDA) with a leave-one-out cross-validation (LOO-CV) procedure. Classification statistics are shown in terms of True Class (TC, i.e. fraction of subjects of each group with a correct classification) and accuracy (i.e. total fraction of subjects with a correct classification). These classification statistics can be presented graphically by pairs, using ROC (Receiving Operating Characteristics) curves (Nakas and Yiannoutsos 2004). In this study, the area under ROC curve (AUC) was used to quantify the ability of the methods to discriminate between pair of groups. Statistically, the value of AUC represents the probability that the measurements of two randomly selected subjects, one from each group, will be properly ordered (Nakas and Yiannoutsos 2004).

Signal processing and statistical analyses were performed using the software packages Matlab (version 7.0; Mathworks, Natick, MA) and SPSS (version 14.0; SPSS Inc, Chicago, IL), respectively.

\section{Results}

\subsection{Spectral entropies}

Initially, three spectral entropies were calculated: SSE, $\operatorname{TSE}(q=2.0)$ and $\operatorname{RSE}(q=3.5)$. Each $10 \mathrm{~s}$ artifact-free MEG segment was analyzed by means of the STFT algorithm, taking non-overlapping windows of $0.5 \mathrm{~s}$. Data were grouped into the five brain areas defined in figure 1. Thus, temporal evolution of parameters was averaged in order to obtain a single entropy value per area and subject. Table 1 summarizes mean entropy values in each region, together with the statistical significance level for pairwise comparisons using Tukey's HSD correction. Figure 2 shows the boxplots corresponding to $\operatorname{SSE}, \operatorname{TSE}(q=$ 2 ) and $\operatorname{RSE}(q=3.5)$ values for each group and region.

The analysis of SSE showed a significant main effect of 'group' variable $(F(1,80)=10.499, p=0.0001)$, but no significant interaction was observed between the variables 'group' and 'region' $(p=0.1054)$. The most significant differences between $\mathrm{AD}$ patients and controls were achieved in the right lateral region $(p<0.0001)$, with similar results in the left lateral region $(p<0.0001)$. The most significant differences between AD and MCI subjects were found in the left lateral region $(p=0.0391)$, with similar results in the posterior $(p=0.0417)$ and anterior $(p=0.0447)$ regions. On the other hand, no significant differences were found between controls and MCI subjects using SSE. 


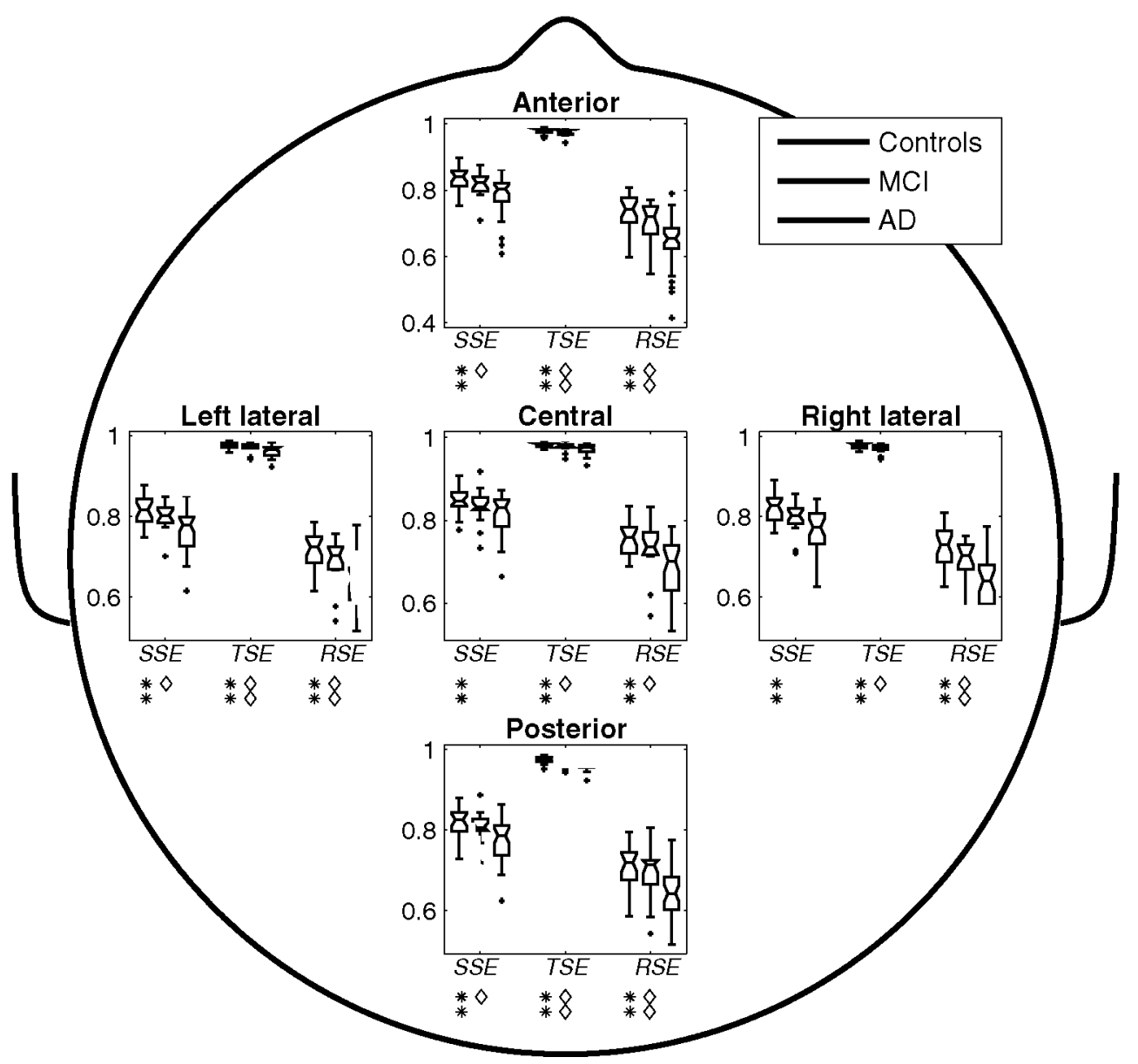

Figure 2. Boxplots representing the distribution of mean values for $\operatorname{SSE}, \operatorname{TSE}(q=2)$ and $\operatorname{RSE}(q=3.5)$ in each region (anterior, central, left lateral, posterior and right lateral). Significant differences between groups are marked with a symbol $\left(^{*} p<0.05\right.$ and ${ }^{* *} p<0.01, \mathrm{AD}$ patients versus controls; $\diamond p<0.05$ and $\diamond \diamond p<0.01$, AD patients versus MCI subjects; $\square p<0.05$ and $\square \square p<0.01$, MCI subjects versus controls).

Table 1. Mean SSE, TSE $(q=2)$ and $\operatorname{RSE}(q=3.5)$ values averaged in each region and for each group, together with the corresponding $p$-values from ANOVA with contrasts.

\begin{tabular}{|c|c|c|c|c|c|c|c|}
\hline \multirow[b]{2}{*}{ Region } & \multirow[b]{2}{*}{ Parameter } & \multicolumn{3}{|c|}{$\mathrm{M} \pm \mathrm{SD}$} & \multicolumn{3}{|c|}{$p$-value } \\
\hline & & $\mathrm{CON}$ & MCI & $\mathrm{AD}$ & $C$ versus $A D$ & $\mathrm{C}$ versus $\mathrm{MCI}$ & $\mathrm{MCI}$ versus $A D$ \\
\hline \multirow[t]{3}{*}{ Anterior } & $\langle\mathrm{SSE}\rangle$ & $0.836 \pm 0.037$ & $0.819 \pm 0.038$ & $0.785 \pm 0.058$ & 0.0001 & 0.3252 & 0.0447 \\
\hline & $\langle\operatorname{TSE}(q=2)\rangle$ & $0.979 \pm 0.007$ & $0.974 \pm 0.010$ & $0.963 \pm 0.019$ & $<0.0001$ & 0.2242 & 0.0077 \\
\hline & $\langle\operatorname{RSE}(q=3.5)\rangle$ & $0.736 \pm 0.054$ & $0.707 \pm 0.059$ & $0.642 \pm 0.074$ & $<0.0001$ & 0.2574 & 0.0023 \\
\hline \multirow[t]{3}{*}{ Central } & $\langle\mathrm{SSE}\rangle$ & $0.847 \pm 0.031$ & $0.834 \pm 0.040$ & $0.815 \pm 0.045$ & 0.0077 & 0.5524 & 0.2312 \\
\hline & $\langle\operatorname{TSE}(q=2)\rangle$ & $0.981 \pm 0.005$ & $0.978 \pm 0.009$ & $0.972 \pm 0.011$ & 0.0001 & 0.4363 & 0.0244 \\
\hline & $\langle\operatorname{RSE}(q=3.5)\rangle$ & $0.756 \pm 0.039$ & $0.732 \pm 0.060$ & $0.687 \pm 0.062$ & $<0.0001$ & 0.3384 & 0.0148 \\
\hline \multirow[t]{3}{*}{ Left lateral } & $\langle\mathrm{SSE}\rangle$ & $0.815 \pm 0.035$ & $0.795 \pm 0.039$ & $0.765 \pm 0.049$ & $<0.0001$ & 0.2190 & 0.0391 \\
\hline & $\langle\operatorname{TSE}(q=2)\rangle$ & $0.976 \pm 0.007$ & $0.971 \pm 0.011$ & $0.961 \pm 0.013$ & $<0.0001$ & 0.1485 & 0.0047 \\
\hline & $\langle\operatorname{RSE}(q=3.5)\rangle$ & $0.717 \pm 0.044$ & $0.690 \pm 0.055$ & $0.635 \pm 0.054$ & $<0.0001$ & 0.1690 & 0.0011 \\
\hline \multirow[t]{3}{*}{ Posterior } & $\langle\mathrm{SSE}\rangle$ & $0.818 \pm 0.039$ & $0.806 \pm 0.041$ & $0.774 \pm 0.050$ & 0.0005 & 0.5732 & 0.0417 \\
\hline & $\langle\operatorname{TSE}(q=2)\rangle$ & $0.975 \pm 0.008$ & $0.972 \pm 0.011$ & $0.963 \pm 0.012$ & 0.0001 & 0.6262 & 0.0084 \\
\hline & $\langle\operatorname{RSE}(q=3.5)\rangle$ & $0.712 \pm 0.053$ & $0.695 \pm 0.063$ & $0.642 \pm 0.054$ & $<0.0001$ & 0.5854 & 0.0040 \\
\hline \multirow{3}{*}{ Right lateral } & $\langle\mathrm{SSE}\rangle$ & $0.822 \pm 0.036$ & $0.795 \pm 0.038$ & $0.767 \pm 0.052$ & $<0.0001$ & 0.0798 & 0.1073 \\
\hline & $\langle\operatorname{TSE}(q=2)\rangle$ & $0.977 \pm 0.007$ & $0.971 \pm 0.010$ & $0.961 \pm 0.014$ & $<0.0001$ & 0.0506 & 0.0149 \\
\hline & $\langle\operatorname{RSE}(q=3.5)\rangle$ & $0.726 \pm 0.046$ & $0.688 \pm 0.056$ & $0.635 \pm 0.061$ & $<0.0001$ & 0.0527 & 0.0046 \\
\hline
\end{tabular}

M: mean. SD: standard deviation. p-value: statistical significance. C: control group. MCI: mild cognitive impairment group. $\mathrm{AD}$ : Alzheimer disease group.

A significant effect of 'group' variable $(F(1,80)=17.392$, $p<0.0001)$, but no significant interaction between 'group' and 'region' variables $(p=0.1402)$, were obtained using
$\operatorname{TSE}(q=2)$. As in the case of SSE, the most significant differences between $\mathrm{AD}$ patients and controls were observed in the right and left lateral regions $(p<0.0001)$, whereas the most 
significant differences between both groups of patients were obtained in the left lateral region $(p=0.0047)$. No statistically significant differences were observed between MCI subjects and controls, though MCI subjects showed a slightly lower $\operatorname{TSE}(q=2)$ value than controls in the right lateral region $(p=0.0506)$.

A significant main effect of 'group' variable $(F(1,80)=$ $19.878, p<0.0001$ ), but no significant interaction between 'group' and 'region' variables ( $p=0.2679)$, were obtained by $\operatorname{RSE}(q=3.5)$. The most significant differences between $\mathrm{AD}$ patients and controls were observed in the right and left lateral regions ( $p<0.0001$ ), whereas the most significant differences between $\mathrm{AD}$ and MCI subjects were found in the left lateral $(p=0.0011)$ and anterior $(p=0.0023)$ regions. On the other hand, no significant differences were observed between MCI subjects and controls, but MCI subjects exhibited a slight decrease of $\operatorname{RSE}(q=3.5)$ in the right lateral region $(p=0.0527)$.

In summary, AD patients showed statistically significant lower entropy values than MCI subjects and controls in almost all comparisons, whereas MCI subjects displayed a slight entropy decrease when compared to controls. These results suggest that dementia progression can be associated with a loss of irregularity in spontaneous MEG activity. The correlation analyses with cognitive and functional tests support this notion. Spectral entropies were positively correlated with MMSE and FAST scores $(p<0.01$ in all regions, except for SSE in the central region where $p<0.05$ ).

\subsection{Disequilibrium}

A significant main effect of 'group' variable was obtained by $\mathrm{ED}(F(1,80)=17.473, p<0.0001)$; however, no interaction between 'group' and 'region' variables was observed using ED $(p=0.1429)$. Table 2 summarizes mean ED values in each region, as well as the $p$-values corresponding to comparisons between groups. Figure 3 shows the distribution of ED values for each region and group. The most significant differences between AD patients and controls were found in the right and left lateral regions $(p<0.0001)$, whereas the most significant differences between both groups of patients were obtained in the left lateral region $(p=0.0047)$. It is noteworthy that significant differences were found between MCI subjects and controls in the right lateral region using ED $(p=0.0487)$, which suggests that MCI subjects exhibit a significant abnormal pattern of disequilibrium in comparison to healthy controls.

In this case, results suggest that dementia progression is accompanied by an increase of disequilibrium in MEG rhythms. This idea is also supported by significant Pearson correlation coefficients between ED and both MMSE and FAST scores ( $p<0.01$ in all regions).

\subsection{Complexity measures}

Finally, three statistical complexity measures (LMC $C_{\text {ED,SSE}}$ $\operatorname{LMC}_{\operatorname{ED}, T S E}(q=2)$ and $\left.\operatorname{LMC} C_{\operatorname{ED}, \operatorname{RSE}(q=3.5)}\right)$ were computed to complement the results provided by the previous parameters. Table 3 summarizes mean statistical complexity values in each region, together with the corresponding $p$-values. Figure 4 shows the distribution of complexity values obtained in each region and for each group. A significant main effect of 'group' variable $(F(1,80)=18.493, p<0.0001)$, but no interaction between 'group' and 'region' variables $(p=0.1611)$, were reached by $\mathrm{LMC}_{\mathrm{ED}, \mathrm{SSE}}$. The most significant differences between $\mathrm{AD}$ patients and controls were found in the right and left lateral regions $(p<0.0001)$, whereas the most significant differences between both groups of patients were found in the left lateral region $(p=0.0028)$. Finally, significant differences between MCI subjects and controls were found in the right lateral region $(p=0.0434)$.

A significant main effect of 'group' variable was obtained by $\operatorname{LMC}_{\mathrm{ED}, \operatorname{TSE}(q=2)}(F(1,80)=17.369, p<0.0001)$; however, no significant interaction between the variables 'group' and 'region' was observed ( $p=0.1470$ ). The most significant differences between AD patients and controls were reached in the right and left lateral regions $(p<0.0001)$, whereas the most significant differences between $\mathrm{AD}$ and $\mathrm{MCI}$ subjects were found in the left lateral region $(p=0.0044)$. No significant differences were observed between MCI subjects and controls, but a slight increase of $\operatorname{LMC} \mathrm{ED,TSE}(q=2)$ for MCI subjects was found in the right lateral region $(p=0.0502)$.

A significant main effect of 'group' variable $(F(1,80)=$ $15.643, p<0.0001$ ), but no significant interaction between the variables 'group' and 'region' ( $p=0.1568$ ), were observed using $\operatorname{LMC}_{\operatorname{ED}, \operatorname{RSE}(q=3.5)}$. The most significant differences between $\mathrm{AD}$ patients and controls were obtained in the right and left lateral regions $(p<0.0001)$, whereas the most significant differences between both groups of patients were reached in the left lateral region $(p=$ 0.0067). No significant differences were found between MCI subjects and controls, though MCI subjects exhibited higher $\mathrm{LMC}_{\mathrm{ED}, \operatorname{RSE}(q=3.5)}$ values than controls in the right lateral region $(p=0.0526)$.

In summary, $\mathrm{AD}$ patients showed significantly higher statistical complexity values than MCI subjects and controls with almost all parameters, whereas MCI subjects displayed significantly higher $\mathrm{LMC}_{\mathrm{ED}, \mathrm{SSE}}$ values than controls. Our findings suggest that dementia progression is associated with an increase in the statistical complexity of spontaneous MEG activity. Moreover, statistically significant correlations were found between the complexity values and both MMSE and FAST scores ( $p<0.01$ in all configurations).

\subsection{Classification analysis}

The statistical analysis was complemented with a classification test in order to evaluate the diagnostic ability of the parameters. Table 4 shows the accuracies and the corresponding AUC values for each pair of groups.

The highest classification rates were achieved when discriminating between $\mathrm{AD}$ patients (identified by the true class $\mathrm{TC}_{\mathrm{AD}}$ ) and controls (identified by the true class $\mathrm{TC}_{\mathrm{CON}}$ ). Thus, the highest AUC values were reached by $\operatorname{RSE}(q=3.5)$ in the left $\left(\mathrm{AUC}=0.890, \mathrm{TC}_{\mathrm{CON}}=80.8 \%, \mathrm{TC}_{\mathrm{AD}}=86.1 \%\right.$, accuracy of $83.9 \%$ ) and right lateral regions $(\mathrm{AUC}=0.880$, $\mathrm{TC}_{\mathrm{CON}}=76.9 \%, \mathrm{TC}_{\mathrm{AD}}=80.6 \%$, accuracy of $\left.79.0 \%\right)$. 


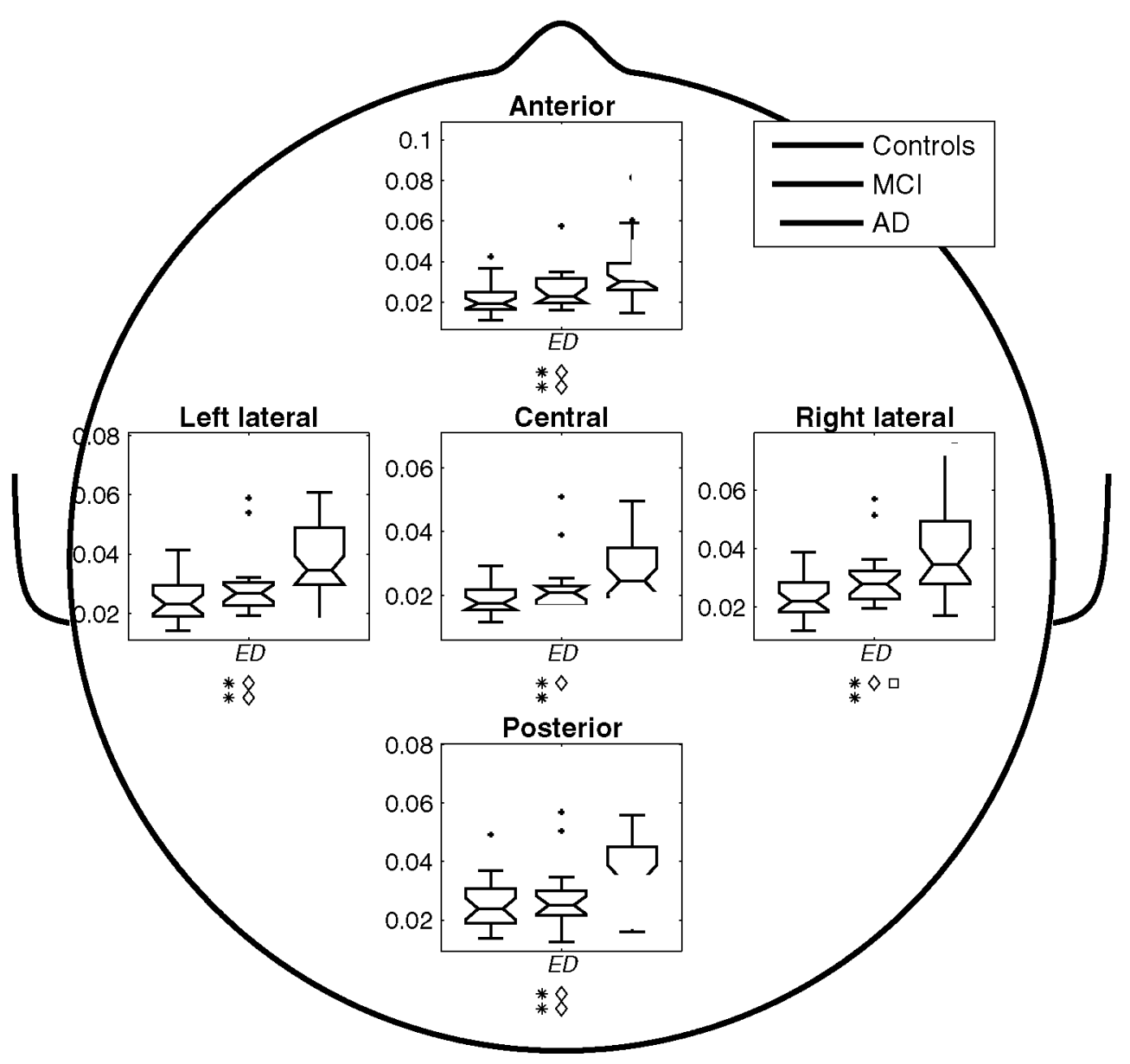

Figure 3. Boxplots representing the distribution of mean values for ED in each region. Significant differences between groups are marked with a symbol $\left({ }^{*} p<0.05\right.$ and ${ }^{* *} p<0.01$, AD patients versus controls; $\diamond p<0.05$ and $\diamond \diamond p<0.01$, AD patients versus MCI subjects; $\square p<0.05$ and $\square \square p<0.01$, MCI subjects versus controls).

Table 2. Mean ED values averaged in each region and for each group, together with the corresponding $p$-values from ANOVA with contrasts.

\begin{tabular}{|c|c|c|c|c|c|c|}
\hline \multirow[b]{2}{*}{ Region } & \multicolumn{3}{|c|}{$\mathrm{M} \pm \mathrm{SD}$} & \multicolumn{3}{|c|}{$p$-value } \\
\hline & $\mathrm{CON}$ & MCI & $\mathrm{AD}$ & $C$ versus $A D$ & $\mathrm{C}$ versus $\mathrm{MCI}$ & $\mathrm{MCI}$ versus $\mathrm{AD}$ \\
\hline Anterior & $0.021 \pm 0.007$ & $0.026 \pm 0.010$ & $0.037 \pm 0.019$ & $<0.0001$ & 0.2159 & 0.0075 \\
\hline Central & $0.019 \pm 0.005$ & $0.022 \pm 0.009$ & $0.028 \pm 0.011$ & 0.0001 & 0.4367 & 0.0240 \\
\hline Left lateral & $0.024 \pm 0.007$ & $0.029 \pm 0.011$ & $0.039 \pm 0.013$ & $<0.0001$ & 0.1450 & 0.0047 \\
\hline Posterior & $0.025 \pm 0.008$ & $0.028 \pm 0.011$ & $0.037 \pm 0.012$ & 0.0001 & 0.6246 & 0.0083 \\
\hline Right lateral & $0.023 \pm 0.007$ & $0.029 \pm 0.010$ & $0.039 \pm 0.014$ & $<0.0001$ & 0.0487 & 0.0152 \\
\hline
\end{tabular}

M: mean. SD: standard deviation. $p$-value: statistical significance. C: control group. MCI: mild cognitive impairment group. AD: Alzheimer disease group.

The classification analysis for MCI (identified by the true class $\mathrm{TC}_{\mathrm{MCI}}$ ) and $\mathrm{AD}$ groups showed that the highest $\mathrm{AUC}$ value was reached using $\operatorname{RSE}(q=3.5)$ in the left lateral region $\left(\mathrm{AUC}=0.810, \mathrm{TC}_{\mathrm{AD}}=61.1 \%, \mathrm{TC}_{\mathrm{MCI}}=88.9 \%\right.$, accuracy of $70.4 \%$ ). The highest accuracy is also achieved by $\operatorname{RSE}(q=3.5)$ in the anterior and posterior regions $\left(\mathrm{TC}_{\mathrm{MCI}}=\right.$ $72.2 \%, \mathrm{TC}_{\mathrm{AD}}=72.2 \%$, accuracy of $72.2 \%$ ).

Finally, the highest AUC values when discriminating between MCI subjects and controls were obtained by $\operatorname{TSE}(q=2), \mathrm{ED}$ and $\mathrm{LMC}_{\mathrm{ED}, \mathrm{SSE}}$ in the right lateral region $\left(\mathrm{AUC}=0.705, \mathrm{TC}_{\mathrm{CON}}=61.5 \%, \mathrm{TC}_{\mathrm{MCI}}=55.6 \%\right.$, accuracy of $59.1 \%$ ). These results agree with the statistical analyses, in which only $\mathrm{ED}$ and $\mathrm{LMC}_{\mathrm{ED}, \mathrm{SSE}}$ displayed significant differences between these two groups. The highest accuracy was obtained using SSE in the anterior (AUC $=0.641$, $\mathrm{TC}_{\mathrm{CON}}=65.4 \%, \mathrm{TC}_{\mathrm{MCI}}=66.7 \%$, accuracy of $\left.65.9 \%\right)$ and right lateral regions $\left(\mathrm{AUC}=0.677, \mathrm{TC}_{\mathrm{CON}}=61.5 \%, \mathrm{TC}_{\mathrm{MCI}}=\right.$ $72.2 \%$, accuracy of $65.9 \%$ ).

\section{Discussion}

We studied the ability of several measures from information theory to characterize spontaneous MEG rhythms from $36 \mathrm{AD}$ patients, $18 \mathrm{MCI}$ subjects and 26 healthy controls. Three spectral entropies (Shannon, Tsallis and Rényi 


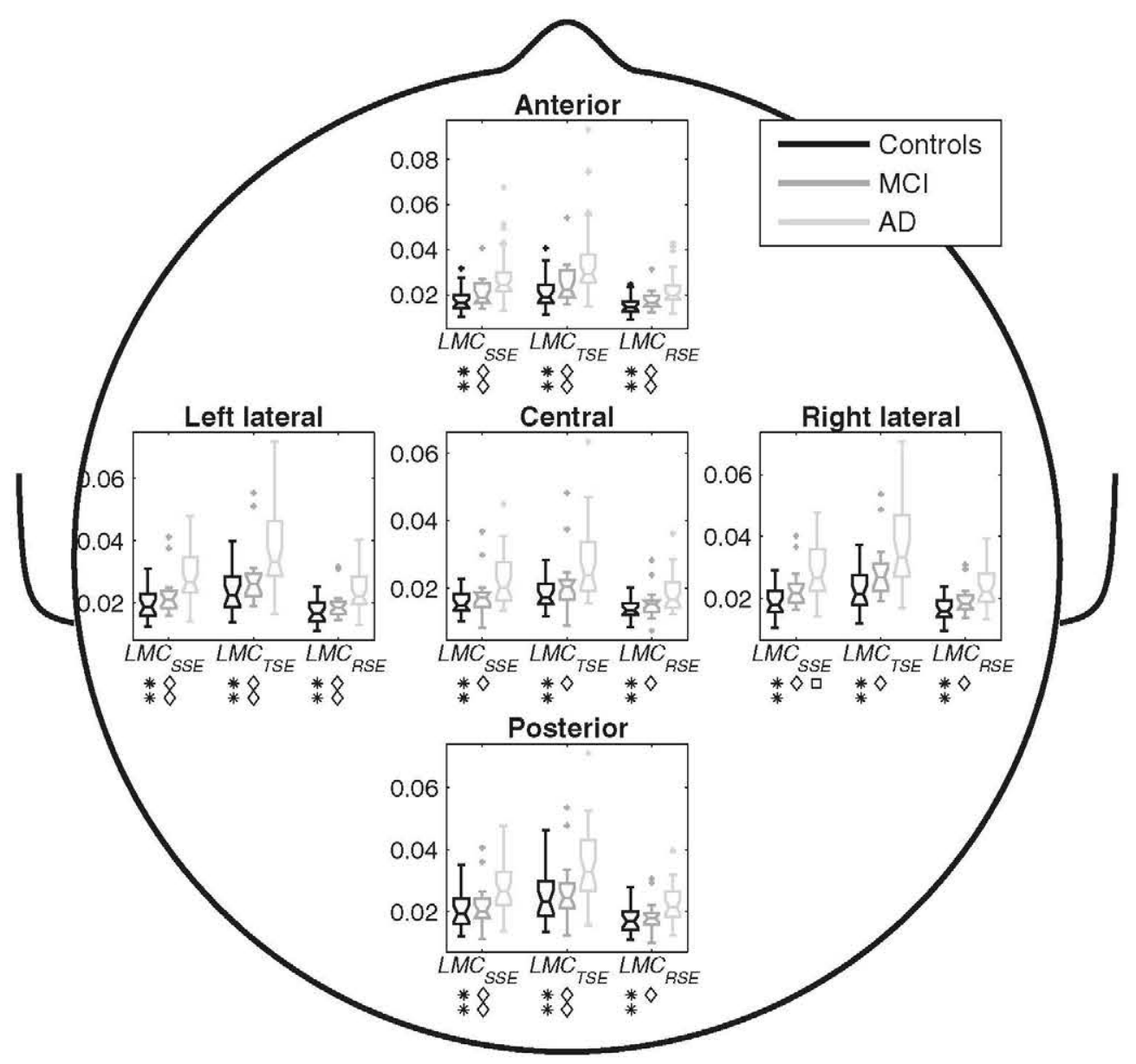

Figure 4. Boxplots representing the distribution of mean values for $\mathrm{LMC}_{\mathrm{SSE}}, \mathrm{LMC}_{\mathrm{TSE}(q=2)}$ and $\mathrm{LMC}_{\mathrm{RSE}(q=3.5) \text {. Significant differences }}$ between groups are marked with a symbol $\left({ }^{*} p<0.05\right.$ and ${ }^{* *} p<0.01$, AD patients versus controls; $\diamond p<0.05$ and $\diamond \diamond p<0.01, \mathrm{AD}$ patients versus MCI subjects; $\square p<0.05$ and $\square \square p<0.01$, MCI subjects versus controls).

Table 3. Mean $\mathrm{LMC}_{\mathrm{ED}, \mathrm{SSE}}, \mathrm{LMC}_{\mathrm{ED}, \mathrm{TSE}(q=2)}$ and $\mathrm{LMC}_{\mathrm{ED}, \mathrm{RSE}(q=3.5)}$ values averaged in each region and for each group, together with the corresponding $p$-values from ANOVA with contrasts.

\begin{tabular}{|c|c|c|c|c|c|c|c|}
\hline \multirow{2}{*}{ Region } & \multirow{2}{*}{ Parameter } & \multicolumn{3}{|c|}{$\mathrm{M} \pm \mathrm{SD}$} & \multicolumn{3}{|c|}{$p$-value } \\
\hline & & CON & MCI & $\mathrm{AD}$ & $\begin{array}{c}C \text { versus } \\
\mathrm{AD}\end{array}$ & $\begin{array}{c}\mathrm{C} \text { versus } \\
\mathrm{MCI}\end{array}$ & $\begin{array}{c}\text { MCI versus } \\
\text { AD }\end{array}$ \\
\hline \multirow[t]{3}{*}{ Anterior } & $\left\langle\mathrm{LMC}_{\mathrm{ED}, \mathrm{SSE}}\right\rangle$ & $0.017 \pm 0.005$ & $0.020 \pm 0.007$ & $0.028 \pm 0.011$ & $<0.0001$ & 0.2037 & 0.0054 \\
\hline & $\left\langle\mathrm{LMC}_{\mathrm{ED}, \mathrm{TSE}(q=2)}\right\rangle$ & $0.021 \pm 0.007$ & $0.025 \pm 0.009$ & $0.035 \pm 0.017$ & $<0.0001$ & 0.2212 & 0.0073 \\
\hline & $\left\langle\operatorname{LMC}_{\mathrm{ED}, \operatorname{RSE}(q=3.5)}\right\rangle$ & $0.015 \pm 0.004$ & $0.017 \pm 0.005$ & $0.022 \pm 0.007$ & $<0.0001$ & 0.2197 & 0.0099 \\
\hline \multirow[t]{3}{*}{ Central } & $\left\langle\mathrm{LMC}_{\mathrm{ED}, \mathrm{SSE}}\right\rangle$ & $0.016 \pm 0.003$ & $0.018 \pm 0.006$ & $0.022 \pm 0.007$ & 0.0001 & 0.4010 & 0.0253 \\
\hline & $\langle\mathrm{LMC}$ ED,TSE $(q=2)\rangle$ & $0.018 \pm 0.005$ & $0.021 \pm 0.009$ & $0.027 \pm 0.010$ & 0.0001 & 0.4284 & 0.0274 \\
\hline & $\left\langle\operatorname{LMC}_{\mathrm{ED}, \operatorname{RSE}(q=3.5)}\right\rangle$ & $0.014 \pm 0.003$ & $0.015 \pm 0.005$ & $0.018 \pm 0.005$ & 0.0003 & 0.4347 & 0.0595 \\
\hline \multirow[t]{3}{*}{ Left lateral } & $\left\langle\mathrm{LMC}_{\mathrm{ED}, \mathrm{SSE}}\right\rangle$ & $0.019 \pm 0.005$ & $0.023 \pm 0.007$ & $0.029 \pm 0.007$ & $<0.0001$ & 0.1422 & 0.0028 \\
\hline & $\left\langle\mathrm{LMC}_{\mathrm{ED}, \mathrm{TSE}(q=2)}\right\rangle$ & $0.024 \pm 0.006$ & $0.028 \pm 0.010$ & $0.037 \pm 0.012$ & $<0.0001$ & 0.1505 & 0.0044 \\
\hline & $\left\langle\mathrm{LMC}_{\mathrm{ED}, \operatorname{RSE}(q=3.5)}\right\rangle$ & $0.017 \pm 0.004$ & $0.019 \pm 0.005$ & $0.024 \pm 0.006$ & $<0.0001$ & 0.1583 & 0.0067 \\
\hline \multirow[t]{3}{*}{ Posterior } & $\left\langle\mathrm{LMC}_{\mathrm{ED}, \mathrm{SSE}}\right\rangle$ & $0.020 \pm 0.006$ & $0.022 \pm 0.007$ & $0.028 \pm 0.007$ & $<0.0001$ & 0.5916 & 0.0071 \\
\hline & $\left\langle\operatorname{LMC}_{\mathrm{ED}, \mathrm{TSE}}(q=2)\right\rangle$ & $0.024 \pm 0.008$ & $0.027 \pm 0.010$ & $0.035 \pm 0.011$ & 0.0001 & 0.6142 & 0.0084 \\
\hline & $\left\langle\mathrm{LMC}_{\mathrm{ED}, \operatorname{RSE}(q=3.5)}\right\rangle$ & $0.017 \pm 0.004$ & $0.019 \pm 0.005$ & $0.023 \pm 0.006$ & 0.0001 & 0.5512 & 0.0125 \\
\hline \multirow[t]{3}{*}{ Right lateral } & $\left\langle\mathrm{LMC}_{\mathrm{ED}, \mathrm{SSE}}\right\rangle$ & $0.019 \pm 0.005$ & $0.023 \pm 0.007$ & $0.029 \pm 0.008$ & $<0.0001$ & 0.0434 & 0.0114 \\
\hline & $\left\langle\mathrm{LMC}_{\mathrm{ED}, \mathrm{TSE}(q=2)}\right\rangle$ & $0.022 \pm 0.006$ & $0.028 \pm 0.010$ & $0.037 \pm 0.012$ & $<0.0001$ & 0.0502 & 0.0149 \\
\hline & $\left\langle\operatorname{LMC}_{\mathrm{ED}, \mathrm{RSE}(q=3.5)}\right\rangle$ & $0.016 \pm 0.004$ & $0.019 \pm 0.005$ & $0.023 \pm 0.006$ & $<0.0001$ & 0.0526 & 0.0256 \\
\hline
\end{tabular}

M: mean. SD: standard deviation. $p$-value: statistical significance. C: control group. MCI: mild cognitive impairment group.

AD: Alzheimer disease group.

entropies), one disequilibrium (Euclidean distance) and three statistical complexities (based on the LMC definition) were calculated using a time-frequency representation to quantify the irregularity and statistical complexity in AD and MCI.
Our findings suggest that dementia progression involves a decrease in irregularity and an increase in both disequilibrium and statistical complexity. Furthermore, our results provide a new interpretation of brain dynamics in $\mathrm{AD}$ and MCI. 
Table 4. Accuracies and AUC values achieved for each parameter, region and pair of groups.

\begin{tabular}{|c|c|c|c|c|c|c|c|}
\hline \multirow[b]{2}{*}{ Region } & \multirow[b]{2}{*}{ Parameter } & \multicolumn{2}{|c|}{$C$ versus $A D$} & \multicolumn{2}{|c|}{$\mathrm{C}$ versus $\mathrm{MCI}$} & \multicolumn{2}{|c|}{ MCI versus $\mathrm{AD}$} \\
\hline & & Accuracy $(\%)$ & AUC & Accuracy $(\%)$ & AUC & Accuracy $(\%)$ & AUC \\
\hline \multirow[t]{7}{*}{ Anterior } & $\langle\mathrm{SSE}\rangle$ & 69.4 & 0.790 & 65.9 & 0.641 & 61.1 & 0.702 \\
\hline & $\langle\operatorname{TSE}(q=2)\rangle$ & 75.8 & 0.856 & 59.1 & 0.647 & 66.7 & 0.756 \\
\hline & $\langle\operatorname{RSE}(q=3.5)\rangle$ & 79.0 & 0.866 & 63.6 & 0.652 & 72.2 & 0.785 \\
\hline & $\langle\mathrm{ED}\rangle$ & 75.8 & 0.856 & 59.1 & 0.647 & 66.7 & 0.756 \\
\hline & $\left\langle\mathrm{LMC}_{\mathrm{ED}, \mathrm{SSE}}\right\rangle$ & 79.0 & 0.860 & 61.4 & 0.650 & 66.7 & 0.769 \\
\hline & $\left\langle\operatorname{LMC}_{\mathrm{ED}, \mathrm{TSE}(q=2)}\right\rangle$ & 75.8 & 0.856 & 59.1 & 0.647 & 66.7 & 0.761 \\
\hline & $\left\langle\mathrm{LMC}_{\mathrm{ED}, \mathrm{RSE}(q=3.5)}\right\rangle$ & 77.4 & 0.845 & 61.4 & 0.643 & 63.0 & 0.750 \\
\hline \multirow[t]{7}{*}{ Central } & $\langle\mathrm{SSE}\rangle$ & 59.7 & 0.704 & 59.1 & 0.622 & 53.7 & 0.590 \\
\hline & $\langle\operatorname{TSE}(q=2)\rangle$ & 69.4 & 0.801 & 61.4 & 0.607 & 61.1 & 0.674 \\
\hline & $\langle\operatorname{RSE}(q=3.5)\rangle$ & 69.4 & 0.809 & 59.1 & 0.603 & 63.0 & 0.691 \\
\hline & $\langle\mathrm{ED}\rangle$ & 69.4 & 0.801 & 61.4 & 0.607 & 61.1 & 0.674 \\
\hline & $\left\langle\mathrm{LMC}_{\mathrm{ED}, \mathrm{SSE}}\right\rangle$ & 64.5 & 0.802 & 61.4 & 0.605 & 61.1 & 0.665 \\
\hline & $\left\langle\mathrm{LMC}_{\mathrm{ED}, \mathrm{TSE}(q=2)}\right\rangle$ & 64.5 & 0.800 & 61.4 & 0.607 & 59.3 & 0.671 \\
\hline & $\left\langle\mathrm{LMC}_{\mathrm{ED}, \operatorname{RSE}(q=3.5)}\right\rangle$ & 62.9 & 0.774 & 61.4 & 0.615 & 61.1 & 0.650 \\
\hline \multirow[t]{7}{*}{ Left lateral } & $\langle\mathrm{SSE}\rangle$ & 66.1 & 0.798 & 56.8 & 0.632 & 61.1 & 0.711 \\
\hline & $\langle\operatorname{TSE}(q=2)\rangle$ & 75.8 & 0.865 & 52.3 & 0.652 & 64.8 & 0.779 \\
\hline & $\langle\operatorname{RSE}(q=3.5)\rangle$ & 83.9 & 0.890 & 52.3 & 0.630 & 70.4 & 0.810 \\
\hline & $\langle E D\rangle$ & 77.4 & 0.865 & 52.3 & 0.652 & 64.8 & 0.779 \\
\hline & $\left\langle\mathrm{LMC}_{\mathrm{ED}, \mathrm{SSE}}\right\rangle$ & 82.3 & 0.875 & 52.3 & 0.650 & 66.7 & 0.792 \\
\hline & $\left\langle\mathrm{LMC}_{\mathrm{ED}, \mathrm{TSE}(q=2)}\right\rangle$ & 75.8 & 0.865 & 52.3 & 0.652 & 64.8 & 0.781 \\
\hline & $\left\langle\operatorname{LMC}_{\mathrm{ED}, \operatorname{RSE}(q=3.5)}\right\rangle$ & 72.6 & 0.845 & 54.6 & 0.652 & 64.8 & 0.769 \\
\hline \multirow[t]{7}{*}{ Posterior } & $\langle\mathrm{SSE}\rangle$ & 62.9 & 0.764 & 61.4 & 0.594 & 64.8 & 0.691 \\
\hline & $\langle\operatorname{TSE}(q=2)\rangle$ & 66.1 & 0.809 & 61.4 & 0.568 & 66.7 & 0.755 \\
\hline & $\langle\operatorname{RSE}(q=3.5)\rangle$ & 74.2 & 0.824 & 50.0 & 0.577 & 72.2 & 0.761 \\
\hline & $\langle\mathrm{ED}\rangle$ & 67.7 & 0.809 & 61.4 & 0.568 & 64.8 & 0.755 \\
\hline & $\left\langle\mathrm{LMC}_{\mathrm{ED}, \mathrm{SSE}}\right\rangle$ & 69.4 & 0.810 & 54.6 & 0.556 & 68.5 & 0.756 \\
\hline & $\left\langle\operatorname{LMC}_{\mathrm{ED}, \mathrm{TSE}(q=2)}\right\rangle$ & 67.7 & 0.809 & 61.4 & 0.571 & 64.8 & 0.755 \\
\hline & $\left\langle\operatorname{LMC}_{\mathrm{ED}, \operatorname{RSE}(q=3.5)}\right\rangle$ & 67.7 & 0.806 & 61.4 & 0.577 & 66.7 & 0.736 \\
\hline \multirow[t]{7}{*}{ Right lateral } & $\langle\mathrm{SSE}\rangle$ & 67.7 & 0.799 & 65.9 & 0.677 & 59.3 & 0.665 \\
\hline & $\langle\mathrm{TSE}(q=2)\rangle$ & 72.6 & 0.855 & 59.1 & 0.705 & 64.8 & 0.730 \\
\hline & $\langle\operatorname{RSE}(q=3.5)\rangle$ & 79.0 & 0.880 & 56.8 & 0.684 & 70.4 & 0.759 \\
\hline & $\langle E D\rangle$ & 71.0 & 0.855 & 59.1 & 0.705 & 64.8 & 0.730 \\
\hline & $\left\langle\mathrm{LMC}_{\mathrm{ED}, \mathrm{SSE}}\right\rangle$ & 72.6 & 0.864 & 59.1 & 0.705 & 66.7 & 0.738 \\
\hline & $\left\langle\operatorname{LMC}_{E D, \operatorname{TSE}(q=2)}\right\rangle$ & 71.0 & 0.853 & 59.1 & 0.701 & 64.8 & 0.736 \\
\hline & $\left\langle\operatorname{LMC}_{\mathrm{ED}, \operatorname{RSE}(q=3.5)}\right\rangle$ & 72.6 & 0.845 & 59.1 & 0.697 & 64.8 & 0.718 \\
\hline
\end{tabular}

AUC: area under ROC curve. C: control group. MCI: mild cognitive impairment group. AD: Alzheimer disease group.

Entropy analysis showed that $\mathrm{AD}$ patients obtained statistically significant lower SSE values than controls $(p<0.01$ in all regions) and MCI subjects $(p<0.05$ in the anterior, left lateral and posterior regions). Similarly, $\operatorname{TSE}(q=$ 2) and $\operatorname{RSE}(q=3.5)$ reached statistically significant lower values for $\mathrm{AD}$ patients than for controls $(p<0.0001$ in all regions) and MCI subjects ( $p<0.05$ in all regions). In addition, MCI subjects displayed slight lower $\operatorname{TSE}(q=$ 2) and $\operatorname{RSE}(q=3.5)$ values than controls in the right lateral region ( $p=0.0506$ and $p=0.0527$, respectively). The entropy decrease suggests that $\mathrm{AD}$ and MCI are accompanied by a loss of frequency components in comparison to normal aging. This result agrees with our previous studies, where a loss of irregularity in AD (Poza et al 2008) and MCI (Bruña et al 2010) was reported. The loss of irregularity can be associated with a decrease in information content (Bezerianos et al 2003). Previous EEG studies identified changes in entropy with a real variation in intra-cortical information flow (Kannathal et al 2005), which leads to suppose that this reduction might involve both a loss of information content
(Baraniuk et al 2001) and an information processing decrease within the brain cortex (Poza et al 2008). As was reported in previous studies (Poza et al 2008), generalized entropies reached greater discrimination statistics than Shannon entropy. Tsallis and Rényi entropies have been used to characterize long and short-range interactions in complex systems, respectively (Bezerianos et al 2003, Stam et al 2006). Therefore, it can be hypothesized that MEG activity in $\mathrm{AD}$ and MCI may be generated by a system in which long and short-range abnormal interactions simultaneously exist.

Statistically significant higher disequilibrium and complexity values were obtained by AD patients when compared to controls ( $p<0.0005$ in all regions) and MCI subjects $(p<0.05$ in all regions, except for $\operatorname{LMC}_{\operatorname{ED}, \operatorname{RSE}(q=3.5)}$ in the central region). It is noteworthy that differences between MCI subjects and controls were statistically significant for ED and $\operatorname{LMC}_{\mathrm{ED}, \mathrm{SSE}}(p<0.05$ in the right lateral region). These results suggest an increase of both disequilibrium and statistical complexity in the early stage of dementia. Similar findings were reported in previous 
studies using disequilibrium (Bruña et al 2010) and neural complexity (van Cappellen van Walsum et al 2003), which lead us to hypothesize that information transmission in the brain becomes more complex from a statistical point of view when dementia progresses. A related idea has been proposed by Drachman (2006), who suggests that cognitive decline in the elderly may be due to complex networks of interacting age-related changes, which may be the fundamental cause in further progression to sporadic AD. Furthermore, the most significant differences between MCI subjects and controls were achieved by statistical complexity measures, which improve the results obtained by spectral entropies and disequilibrium. Therefore, these measures might reflect some underlying characteristics of MEG activity from MCI subjects that cannot be detected independently by the irregularity and disequilibrium parameters.

Some discussion about the complexity increase is required. Previous MEG studies used linear and nonlinear measures to quantify the irregularity and complexity patterns in $\mathrm{AD}$. Their results suggested that dementia is characterized by a loss in irregularity (Poza et al 2007, 2008, Hornero et al 2009) and complexity (Gómez et al 2009a, Hornero et al 2009). Previous studies based on Lempel-Ziv complexity showed that MCI is related to a loss of irregularity in MEG rhythms (Fernández et al 2010). Our findings support the notion that both $\mathrm{AD}$ and $\mathrm{MCI}$ elicit a decrease in irregularity, though an increase of statistical complexity is also observed. Certainly, irregularity and complexity parameters are linked to some extent. They quantify the degree of disorder in brain dynamics, focusing on different characteristics. Therefore, they are useful to provide a statistical description of the variability within the MEG or a measure of average uncertainty of signal information (Bezerianos et al 2003, Kannathal et al 2005). On the other hand, statistical complexity measures are clearly dissociated from this interpretation of complexity. They identify highly regular or irregular signals as trivial states, since they are both considered as not very informative (total order or perfect randomness). As a consequence, their complexity is zero (López-Ruiz et al 1995). Signals of high statistical complexity (and therefore highly informative) should be generated by a system where an optimal balance between a completely independent and a totally dependent functioning of their elementary components can be found (Sporns et al 2000). $\mathrm{LMC}$ is a new approach in that direction, such that it is expected to provide a novel quantitative strategy for assessing complexity patterns in brain dynamics. Increasing LMC values suggest that MEG rhythms are generated by a system where a balanced interplay between dependent and independent functioning can be found. There is no evidence that a high LMC value necessarily reflects an optimal information processing, but it suggests some kind of highly informative processing (in terms of statistical complexity). On the basis of the previous ideas, the statistical complexity exhibits a convex shape when it is plotted against the irregularity, as depicted in figure 5 .

$\mathrm{AD}$ has been described as a disconnection syndrome, where functional interactions in the brain are strongly affected by anatomical abnormalities among different cortical areas (Jelles et al 1999) and altered cholinergic coupling interactions

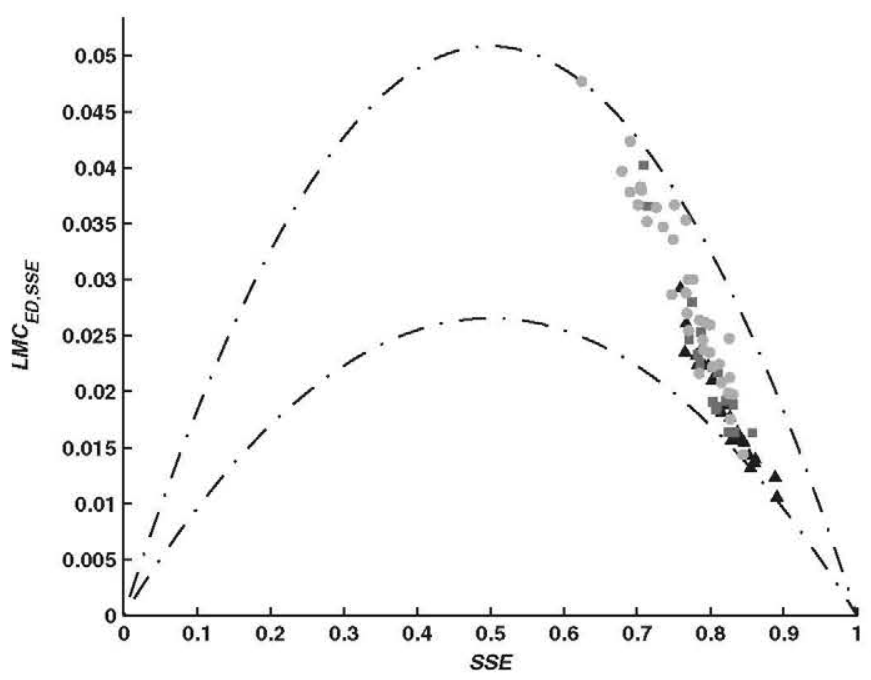

Figure 5. Relationship between $\mathrm{LMC}_{\mathrm{SSE}}$ and $\mathrm{SSE}$ in the right lateral region for each group ( $\boldsymbol{\Lambda}$ : Controls; $\mathbf{\square}$ : MCI subjects; $\bullet$ : AD patients). The maximum and minimum $\mathrm{LMC}_{\mathrm{SSE}}$ values as a function of SSE are depicted by chain lines.

among cortical neurons (Jeong 2004). Recent studies pose the hypothesis that AD is characterized by more evident statistical deterministic relationships in MEG connectivity (Alonso et al 2011). Our findings also support this idea. Some researchers suggest that the loss of complexity, usually associated to $\mathrm{AD}$, may be influenced by both increases and decreases of functional couplings in different frequency bands (van Cappellen van Walsum et al 2003, Stam et al 2006, 2007, 2009, Alonso et al 2011). Previous studies reported that functional connectivity in $\mathrm{AD}$ increases in low-frequency bands (Locatelli et al 1998, Montez et al 2009, Alonso et al 2011) and decreases in high-frequency bands (Stam et al 2007 , 2009). These connectivity patterns have been associated with the impairment that AD causes in brain networks (Locatelli et al 1998, Stam et al 2009) or with the activation of some kind of compensatory mechanism to overcome the structural and functional brain deficits elicited by dementia (Stam 2010). A possible explanation to the increase of statistical complexity in $\mathrm{AD}$ and MCI might be such compensatory functional response. There exists evidence of compensatory mechanisms by which preclinical $\mathrm{AD}$ brain attempts to compensate for neurodegeneration, before the manifestation of cognitive and functional impairments (Becker et al 1996).

Another hypothesis to explain the increase of complexity could be that entropy quantifiers overestimate the irregularity of the system. Spectral entropies are intrinsically related to the concept of a signal component, so that signals constructed from a reduced number of elementary components are supposed to yield low entropy values (Baraniuk et al 2001). Nevertheless, different SSE values are observed in $\mathrm{AD}$ patients and controls depending on the nature of the recording. EEG studies obtained lower SSE values than those reported by MEG studies (Abásolo et al 2006, Poza et al 2008). EEG and MEG reflect slightly different characteristics of neuronal activity (Rampp and Stefan 2007). Therefore, brain dynamics are expected to elicit different irregularity patterns. In this context, entropybased measures, such as spectral entropies, could provide 
different estimates of the degree of disorder in the brain, which might influence the interpretation in terms of irregularity. As a consequence, it could be interesting to apply different entropy definitions, such as nonlinear ones, in order to explore new LMC formulations. Future analyses should address these concerns to further assess the role of entropy-based measures in the quantification of EEG and MEG irregularity.

Further evidence on the discrepancies between EEG and MEG has been reported by Stam and co-workers in several studies (Stam et al 2007, 2009). They analyzed the relationship between complexity and functional connectivity in $\mathrm{AD}$ by means of synchronization measures. Concepts from graph theory were used to quantify the characteristics of functional connectivity networks. Although similar results were obtained in EEG and MEG studies, some differences were observed in the calculation of the clustering coefficient (a parameter that quantifies the local connectedness of a network). Researchers suggested that differences could be due to volume conduction effects, which produced high estimates of the clustering coefficient in EEG analyses (Stam et al 2009). As reported in previous studies, they also pointed out that AD can be associated with a loss of resting-state functional connectivity in alpha (Stam et al 2009) and beta bands (Stam et al 2007, 2009). Furthermore, they observed that AD is characterized by a more random, but less complex, large-scale topology of functional connectivity networks than normal aging (Stam et al 2009). In this context, the notion of complexity differs from the nonlinear and the statistical definitions, since Stam et al (2007, 2009) refer to the complexity of patterns of interrelations. This fact stresses the importance of complexity as a context-dependent parameter that can summarize different (but complementary) features of the underlying brain dynamics.

MCI is usually considered as a pre-clinical stage of AD (Petersen 2009). Thus, an early AD diagnosis should be established on the basis of a previous MCI detection. Our findings suggest that MCI exhibit intermediate alterations between $\mathrm{AD}$ and normal aging, which agrees with the results of previous research (Fernández et al 2006, 2010, Escudero et al 2011). Previous MEG studies reported classification rates of $\mathrm{AD}$ patients versus elderly controls about $80 \%$ (Fernández et al 2006, Poza et al 2007, 2008, Gómez et al 2009a, Hornero et al 2009, Stam 2010, Alonso et al 2011, Escudero et al 2011). Our classification results are similar to this value, since the highest accuracy was $83.9 \%$. On the other hand, previous MEG studies usually reached an accuracy about $65 \%$ to discriminate between MCI and control subjects (Fernández et al 2006, 2010, Gómez et al 2009a, Escudero et al 2011). Our highest accuracy was $65.9 \%$. It is worth noting that our classification statistics are comparable to those reported in previous MEG studies, though they did not use any LOO-CV procedure (Fernández et al 2006, 2010, Gómez et al 2009b). The LOO-CV procedure reduces the accuracy, but it provides an almost unbiased estimate of the true generalization ability of the classification model and avoids over-estimation of the true classification rates (Escudero et al 2011).

Finally, a number of methodological and clinical issues merit further consideration. The spectral analysis was accomplished using the STFT. However, other time-frequency representations, such as wavelets (Wan et al 2006), could be also considered to compute entropies, disequilibrium and statistical complexities. It is worth noting that the choice of the STFT was based on several premises: (i) the STFT provides a well-established framework that eases the interpretation of spectral parameters; (ii) the STFT has been widely applied to characterize EEG/MEG activity (Jeong 2004, Stam 2010) and previous MEG studies have shown its usefulness in describing spontaneous brain activity in dementia (Poza et al 2008); and (iii) previous MEG investigations have shown that the proposed parameters are not strongly influenced by the spectral estimation (Poza and Hornero 2011). Other possible comment is related to the choice of the entropic index $q$ in the parameterized entropies (TSE and RSE). Different entropic indexes correspond to particular statistical mechanics. Other $q$ values could lead to different complexity patterns that might be useful to characterize MCI. Nevertheless, several assumptions, based on physiological and methodological evidences, were made to carefully select the entropic indexes. On the basis of previous studies (Tong et al 2002, Rosso et al 2006), the brain activity was modeled as a sub-extensive system $(q>1)$. Lower $q$ values are rather difficult to justify and have not been recommended for clinical purposes (Rosso et al 2006). Likewise, the role of the entropic index $(q>1)$ has been found to be relevant to characterize MEG activity in AD (Poza et al 2008). The results in this study suggested that TSE was sensitive to the parameter $q$ (due to its exponential definition), whereas RSE showed little dependence on the entropic index (due to its logarithmic definition) (Poza et al 2008). In the present study, the values that exhibited the highest performance to characterize $\mathrm{AD}$ were selected (i.e. $q=2$ and $q=3.5$ for TSE and RSE, respectively). Thus, a direct comparison with previous research can be easily made. It should be pointed out that other neurodegenerative diseases also elicit abnormal irregularity and complexity patterns in EEG and MEG activity. Further research should be carried out to compare the patterns associated to each disorder. Besides, despite the high progression rate from MCI to dementia, MCI is actually a heterogeneous disorder, which does not necessarily result in $\mathrm{AD}$. Therefore, a longitudinal analysis could be useful to analyze MCI progression.

\section{Conclusions}

In summary, our findings suggest that MCI exhibit intermediate alterations, in the irregularity and statistical complexity patterns, between $\mathrm{AD}$ and normal aging. Our results support the notion that spectral entropies, disequilibrium and statistical complexity measures may lead to a better understanding of the underlying brain dynamics in $\mathrm{AD}$ and MCI. Furthermore, these new measures extend the concept of complexity and provide useful descriptors of spontaneous MEG rhythms in these neuropathologies.

Future efforts will be addressed to explore other irregularity and disequilibrium measures, useful to characterize MEG rhythms in AD and MCI. Additionally, further work should be attempted in order to extend the results to other dementias. 


\section{Acknowledgments}

The authors would like to thank the 'Asociacion de Familiares de Enfermos de Alzheimer' (AFAL) and the Geriatric Unit of the 'Hospital Clínico Universitario San Carlos' for supplying the subjects who participated in this study. They are also thankful to María Gil and Beatriz RodríguezBatllori, who revised the English version of this paper. Likewise, the authors are grateful to the reviewers and the editorial office for their feedback about the manuscript. This work has been partially supported by the following contract grant sponsors: Subdirección General de Proyectos de Investigación, Ministerio de Ciencia e Innovación, Spain, Contract grant number TEC2011-22987; and Fundación General CSIC, 'Proyectos Cero on Ageing', Spain.

Abásolo D, Hornero R, Espino P, Álvarez D and Poza J 2006 Entropy analysis of the EEG background activity in Alzheimer's disease patients Physiol. Meas. 27 241-53

Alonso J F, Poza J, Mañanas M A, Romero S, Fernández A and Hornero R 2011 MEG connectivity analysis in patients with Alzheimer's disease using cross mutual information and spectral coherence Ann. Biomed. Eng. 39 524-36

Baraniuk R G, Flandrin P, Janssen A J E M and Michel O J J 2001 Measuring time-frequency information content using the Rényi entropies IEEE Trans. Inf. Theory 47 1391-409

Becker J T, Mintun M A, Aleva K, Wiseman M B, Nichols T and DeKosky S T 1996 Compensatory reallocation of brain resources supporting verbal episodic memory in Alzheimer's disease Neurology 46 692-700 PMID: 8618669

Berendse H W, Verbunt J P A, Scheltens Ph, van Dijk B W and Jonkman E J 2000 Magnetoencephalographic analysis of cortical activity in Alzheimer's disease: a pilot study Clin. Neurophysiol. 111 604-12

Bezerianos A, Tong S and Thakor N 2003 Time-dependent entropy estimation of EEG rhythm changes following brain ischemia Ann. Biomed. Eng. 31 221-32

Blennow K, de Leon M J and Zetterberg H 2006 Alzheimer's disease Lancet 368 387-403

Bruña R, Poza J, Gómez C, Fernández A and Hornero R 2010 Analysis of spontaneous MEG activity in mild cognitive impairment using spectral entropies and disequilibrium measures Conf. Proc. IEEE Eng. Med. Biol. Soc. (Buenos Aires, Argentina, 31 August 4 September 2010) pp 6296-9 PMID: 21097360

Cummings J L 2004 Alzheimer's disease N. Engl. J. Med. $35156-67$

Drachman D A 2006 Aging of the brain, entropy, and Alzheimer disease Neurology 67 1340-52

Escudero J, Sanei S, Jarchi D, Abásolo D and Hornero R 2011 Regional coherence evaluation in mild cognitive impairment and Alzheimer's disease based on adaptively extracted magnetoencephalogram rhythms Physiol. Meas. $321163-80$

Fernández A, Hornero R, Mayo A, Poza J, Gil-Gregorio P and Ortiz T 2006 MEG spectral profile in Alzheimer's disease and mild cognitive impairment Clin. Neurophysiol. $117306-14$

Fernández A, Hornero R, Gómez C, Turrero A, Gil-Gregorio P, Matías-Santos J and Ortiz T 2010 Complexity analysis of spontaneous brain activity in Alzheimer's disease and mild cognitive impairment: an MEG study Alzheimer Dis. Assoc. Disord. 24 182-9
Georgopoulos A P et al 2007 Synchronous neural interactions assessed by magnetoencephalography: a functional biomarker for brain disorders J. Neural Eng. 4 349-55

Gómez C, Hornero R, Abásolo D, Fernández A and Escudero J 2009a Analysis of MEG background activity in Alzheimer's disease using non-linear methods and ANFIS Ann. Biomed. Eng. 37 586-94

Gómez C, Stam C J, Hornero R, Fernández A and Maestú F 2009b Disturbed beta band functional connectivity in patients with mild cognitive impairment: a MEG study IEEE Trans. Biomed. Eng. 56 1683-90

Hornero R, Abásolo D, Escudero J and Gómez C 2009 Nonlinear analysis of electroencephalogram and magnetoencephalogram recordings in patients with Alzheimer's disease Phil. Trans. A $367317-36$

Inouye T, Shinosaki K, Sakamoto H, Toi S, Ukai S, Iyama A, Katsuda Y and Hirano M 1991 Quantification of EEG irregularity by use of the entropy of the power spectrum Electroencephalogr. Clin. Neurophysiol. 79 204-10

Jalbert J J, Daiello L A and Lapane K L 2008 Dementia of the Alzheimer type Epidemiol. Rev. 30 15-34

Jelles B, van Birgelen J H, Slaets J P, Hekster R E, Jonkman E J and Stam C J 1999 Decrease of non-linear structure in the EEG of Alzheimer patients compared to healthy controls Clin. Neurophysiol. 110 1159-67

Jeong J 2004 EEG dynamics in patients with Alzheimer's disease Clin. Neurophysiol. 115 1490-505

Kannathal N, Choo M L, Acharya U R and Sadasivan P K 2005 Entropies for detection of epilepsy in EEG Comput. Methods Programs Biomed. 80 187-94

Locatelli T, Cursi M, Liberati D, Franceschi M and Comi G 1998 EEG coherence in Alzheimer's disease Electroencephalogr. Clin. Neurophysiol. 106 229-37

López-Ruiz R, Mancini H L and Calbet X 1995 A statistical measure of complexity Phys. Lett. A 209 321-6

Martin M T, Plastino A and Rosso O A 2003 Statistical complexity and disequilibrium Phys. Lett. A 311 126-32

McKhann G, Drachman D, Folstein M, Katzman R, Price D and Stadlan E M 1984 Clinical diagnosis of Alzheimer's disease: report of the NINCDS-ADRDA work group under the auspices of Department of Health and Human Services Task Force on Alzheimer's disease Neurology 34 939-44 http://neurology.org/content/34/7/939.abstract

Minati L, Edginton T, Bruzzone M G and Giaccone G 2009 Current concepts in Alzheimer's disease: a multidisciplinary review Am. J. Alzheimers Dis. Other Demen. 24 95-121

Montez T et al 2009 Altered temporal correlations in parietal alpha and prefrontal theta oscillations in early-stage Alzheimer disease Proc. Natl Acad. Sci. USA 106 1614-9

Nakas C T and Yiannoutsos C T 2004 Ordered multiple-class ROC analysis with continuous measurements Stat. Med. 23 3437-49

Nunez P L, Wingeier B M and Silberstein R B 2001 Spatial-temporal structures of human alpha rhythms: microcurrents sources, multiscale elements, and global binding of local networks Hum. Brain Mapp. 13 125-64

Osipova D, Rantanen K, Ahveninen J, Ylikoski R, Happola O, Strandberg T and Pekkonen E 2006 Source estimation of spontaneous MEG oscillations in mild cognitive impairment Neurosci. Lett. 405 57-61

Petersen R C 2009 Early diagnosis of Alzheimer's disease: Is MCI too late? Curr. Alzheimer Res. 6 324-30

Petersen R C, Doody R, Kurz A, Mohs R C, Morris J C, Rabins P V, Ritchie K, Rossor M, Thal L and Winblad B 2001 Current concepts in mild cognitive impairment Arch. Neurol. $\mathbf{5 8}$ 1985-92

Petersen R C, Roberts R O, Knopman D S, Boeve B F, Geda Y E, Ivnik R J, Smith G E and Jack C R Jr 2009 Mild cognitive impairment: ten years later Arch. Neurol. 66 1447-55

Poza J and Hornero R 2011 Time-frequency analysis of MEG activity in Alzheimer's disease Recent Advances in Biomedical 
Signal Processing ed J M Górriz, E W Lang and J Ramírez (Bentham: eBook) pp 123-40

Poza J, Hornero R, Abásolo D, Fernández A and García M 2007 Extraction of spectral based measures from MEG background oscillations in Alzheimer's disease Med. Eng. Phys. 29 1073-83

Poza J, Hornero R, Escudero J, Fernández A and Sánchez C I 2008 Regional analysis of spontaneous MEG rhythms in patients with Alzheimer's disease using spectral entropies Ann. Biomed. Eng. 36 141-52

Rampp S and Stefan H 2007 On the opposition of EEG and MEG Clin. Neurophysiol. $1181658-9$

Rényi A 1970 Probability Theory (Amsterdam: North-Holland)

Rosso O A, Martin M T, Figliola A, Keller K and Plastino A 2006 EEG analysis using wavelet-based information tools J. Neurosci. Methods $153163-82$

Sporns O, Tononi G and Edelman G M 2000 Connectivity and complexity: the relation between neuroanatomy and brain dynamics Neural Netw. $13909-22$

Stam C J 2005 Nonlinear dynamical analysis of EEG and MEG: review of an emerging field Clin. Neurophysiol. $1162266-301$

Stam C J 2010 Use of magnetoencephalography (MEG) to study functional brain networks in neurodegenerative disorders J. Neurol. Sci. 289 128-34
Stam C J, Jones B F, Manshanden I, van Cappellen van Walsum A M, Montez T, Verbunt J P, de Munck J C, van Dijk B W, Berendse H W and Scheltens P 2006 Magnetoencephalographic evaluation of resting-state functional connectivity in Alzheimer's disease Neuroimage 32 1335-44

Stam C J, Jones B F, Nolte G, Breakspear M and Scheltens P 2007 Small-world networks and functional connectivity in Alzheimer's disease Cereb. Cortex. 17 92-9

Stam C J et al 2009 Graph theoretical analysis of magnetoencephalographic functional connectivity in Alzheimer's disease Brain 132 213-24

Tong S, Bezerianos A, Paul J, Zhu Y and Thakor N 2002 Nonextensive entropy measure of EEG following brain injury from cardiac arrest Physica A $\mathbf{3 0 5} 619-28$

Tsallis C 1988 Possible generalization of Boltzmann-Gibbs statistics J. Stat. Phys. 52 479-87

van Cappellen van Walsum A M, Pijnenburg Y A L, Berendse H W, van Dijk B W, Know D L, Scheltens Ph and Stam C J 2003 A neural complexity measure applied to MEG data in Alzheimer's disease Clin. Neurophysiol. 114 1034-40

Wan B, Ming D, Fu X, Yang C, Qi H and Chen B 2006 Study on a quantitative electroencephalography power spectrum typical of Chinese Han Alzheimer's disease patients by using wavelet transforms J. Neural Eng. 3 71-7 